Disentangling overlapping high-field EPR spectra of organic radicals: Identification of light-induced polarons in the record fullerene-free solar cell blend PBDB-T:ITIC

Peer-reviewed author version

VAN LANDEGHEM, Melissa; MAES, Wouter; Goovaerts, Etienne \& VAN

DOORSLAER, Sabine (2018) Disentangling overlapping high-field EPR spectra of organic radicals: Identification of light-induced polarons in the record fullerene-free solar cell blend PBDB-T:ITIC. In: JOURNAL OF MAGNETIC RESONANCE, 288, p. 1-10.

DOI: 10.1016/j.jmr.2018.01.007

Handle: http://hdl.handle.net/1942/26243 


\title{
Disentangling overlapping high-field EPR spectra of organic radicals: Identification of light-induced polarons in the record fullerene-free solar cell blend PBDB-T:ITIC
}

\author{
Melissa Van Landeghem ${ }^{\mathrm{a}}$, Wouter Maes ${ }^{\mathrm{b}}$, Etienne Goovaerts $^{\mathrm{a}, *}$, Sabine Van Doorslaer ${ }^{\mathrm{a}, *}$ \\ ${ }^{a}$ Department of Physics, University of Antwerp, Universiteitsplein 1, 2610 Antwerpen, Belgium \\ ${ }^{b}$ Institute for Materials Research, Design $\&$ Synthesis of Organic Semiconductors, Hasselt University, Agoralaan 1, 3590 \\ Diepenbeek, Belgium
}

\begin{abstract}
We present a combined high-field EPR and DFT study of light-induced radicals in the bulk heterojunction blend of PBDB-T:ITIC, currently one of the highest efficiency non-fullerene donor:acceptor combinations in organic photovoltaics. We demonstrate two different approaches for disentangling the strongly overlapping high-field EPR spectra of the positive and negative polarons after charge separation: (1) relaxation-filtered field-swept EPR based on the difference in $T_{1}$ spin-relaxation times and (2) field-swept EDNMR-induced EPR by exploiting the presence of ${ }^{14} \mathrm{~N}$ hyperfine couplings in only one of the radical species, the small molecule acceptor radical. The approach is validated by light-induced EPR spectra on related blends and the spectral assignment is underpinned by DFT computations. The broader applicability of the spectral disentangling methods is discussed.
\end{abstract}

\section{Introduction}

Solution-processed organic solar cells (OSCs) are considered a highly promising technology due to their low manufacturing costs and compatibility with flexible substrates allowing for innovative photovoltaic applications. For two decades, the research efforts into increasing the power conversion efficiencies (PCEs) of OSCs mainly relied on bulk heterojunction (BHJ) blends of a conjugated polymer and a fullerene acceptor such as [6,6]-phenyl- $\mathrm{C}_{61}$-butyric acid methyl ester (PCBM). In the last few years, however, OSCs with non-fullerene acceptors have attracted considerable research interest due to their enhanced absorptivity and chemical tunability as compared to conventional fullerenes [1-3]. Careful molecular design of the acceptor materials allows OSCs to surpass the $11.7 \%$ certified record efficiency of singlejunction polymer-fullerene solar cells [4]. The donor-acceptor combination studied in this work, poly[(2,6-(4,8-bis(5-(2-ethylhexyl)thiophen-2-yl)-

\footnotetext{
* Corresponding author

Email addresses:

melissa.vanlandeghem@uantwerpen. be (Melissa Van Landeghem), wouter.maes@uhasselt.be (Wouter Maes), etienne.goovaerts@uantwerpen.be (Etienne Goovaerts), sabine.vandoorslaer@uantwerpen. be (Sabine Van Doorslaer)
}

benzo[1,2- $\left.b: 4,5-b^{\prime}\right]$ dithiophene $\left.)\right)$-alt-(5,5-(1', 3'di-2-thienyl-5',7'-bis(2-ethylhexyl)benzo[1',2'$c: 4^{\prime}, 5$ '-c']dithiophene-4,8-dione))] (PBDB-T) with 3,9-bis(2-methylene-(3-(1,1-dicyanomethylene)indanone) )-5,5,11,11-tetrakis(4-hexylphenyl)dithieno[2,3- $d: 2^{\prime}, 3^{\prime}$ - $\left.d^{\prime}\right]$ - $s$-indaceno[1,2- $\left.b: 5,6-b^{\prime}\right]$ dithiophene (ITIC), was the first fullerene-free OSC to recently achieve $>11 \%$ efficiency, challenging the state-of-the-art polymer- $\mathrm{PC}_{71} \mathrm{BM}$ devices $[5,6]$. To the best of our knowledge, this work presents the first EPR characterization of charge-transfer radicals in a blend of PBDB-T and ITIC.

Charge transfer and separation in the donoracceptor contact regions are essential steps of the photovoltaic process in BHJ OSCs, which lead to the production of positive and negative free charges, called polarons. These are radical states in the blend localized on the donor and acceptor molecules, respectively. Also other paramagnetic species, such as coupled radical pairs and triplet excitons are important for understanding of the device operation and optimization of its efficiency. Over the past decades, light-induced EPR has played a crucial role in the study of OSCs because of its selectiveness in detecting the different paramagnetic species created in the BHJ blend under illumination. As such, EPR can distinguish between polarons in the donor or acceptor regions. Characterization of the photogenerated charge car- 
riers in terms of their $\boldsymbol{g}$-tensor and hyperfine couplings with surrounding magnetic nuclei reveals detailed information on the electronic structure and delocalization of the polarons throughout the organic semiconductor, especially when combined with quantum-chemical calculations for the interpretation of the magnetic resonance parameters $[7,8]$. Knowledge of these properties is key to understand the efficiency gains in novel OSC blends. As recent studies suggest, material design towards extended delocalization of the charge carriers is essential to ensure efficient charge separation [9] and to avoid unwanted population of low-lying triplet states resulting in photocurrent losses [10]. This is especially relevant considering the current efforts in enhancing the open circuit voltage $\left(\mathrm{V}_{\mathrm{oc}}\right)$ of the solar cells by minimizing the energy level offsets between donor and acceptor, thereby lowering the driving force for exciton dissociation $[6,11,12]$. Besides the unique ability of EPR to directly probe the electronic structure and charge delocalization, dedicated EPR techniques have been applied to access other types of information, including transient EPR for the study of charge generation dynamics $[13,14]$, optically detected magnetic resonance (ODMR) to unravel detrimental triplet formation pathways [15-17], and electrically detected magnetic resonance (EDMR) for transport-related mechanisms in devices $[15,16,18]$.

Since light-induced radicals in OSC blends are always created in pairs by exciton dissociation, often the problem of overlapping EPR spectra arises [8, 19-21]. Usually one then resorts to highfrequency EPR where the increased spectral resolution allows for identification of the radicals based on their effective $g$-values $[8,22,23]$. However, in the case of PBDB-T:ITIC, we could not separate the two-component structure of the spectrum even at $\mathrm{W}$-band frequency $(94 \mathrm{GHz})$. Therefore we separated the two contributions to the total EPR spectrum exploiting two different properties of the radicals, namely the (small) difference in their longitudinal $\left(T_{1}\right)$ relaxation times and the presence of nitrogen atoms, and therefore ${ }^{14} \mathrm{~N}$ hyperfine couplings, in the ITIC molecule, which are absent in the polymer.

To exploit the difference in $T_{1}$ times, we applied an inversion-recovery filter to selectively suppress one component similar to the relaxation-filtered hyperfine spectroscopy (REFINE) technique proposed by Maly et al. [24, 25]. The viability of this technique to separate individual contributions to fieldswept EPR spectra has already been demonstrated on a mixture of two organic radicals and a $\mathrm{Cu}(\mathrm{II})$ complex by Cernescu et al. [26]. Moreover, the authors claimed that this relaxation-filtered tech- nique can be applied to a two-component mixture with $T_{1}$ ratios as small as $1: 2$, rendering it generally applicable to most overlapping spectra encountered in (bio)organic and (bio)inorganic systems.

Sensitive detection of the ${ }^{14} \mathrm{~N}$ hyperfine couplings at W-band frequency was achieved by means of electron-electron double resonance (ELDOR)detected NMR (EDNMR). This double-resonance technique, first described by Schosseler et al. [27], employs a high turning angle (HTA) preparation pulse at microwave (mw) frequency $\nu_{1}$ to selectively excite forbidden EPR transitions, resulting in a polarization transfer from an allowed EPR transition to the corresponding nuclear transition. The EDNMR spectrum is obtained by sweeping the HTA frequency about the resonant frequency $\nu_{0}$ of the detection sequence (Figure 2(b)). Whenever $\Delta \nu=\nu_{1}-\nu_{0}$ matches a nuclear frequency of the system, a reduction of the detected EPR intensity is observed $[27,28]$. The main drawback of EDNMR is the central blind spot occurring when $\nu_{1}=\nu_{0}$, which could possibly mask those spinforbidden transitions close in frequency to the allowed EPR transitions. Therefore EDNMR is most effective at high fields when the spectral positions of weakly coupled nuclei, centered about their respective Larmor frequencies, shift away from the central blind spot $[29,30]$. In this work the EDNMR pulse sequence with the HTA frequency offset $\Delta \nu$ fixed on resonance with the ${ }^{14} \mathrm{~N}$ hyperfine couplings is applied to obtain an EDNMRinduced field-swept EPR spectrum containing only contributions from the ITIC radical. This is to our knowledge the first demonstration of EDNMRinduced EPR.

In this work a full EPR characterization of the charge-separated states in the high performance solar cell blend PBDB-T:ITIC is presented. We adress the problem of overlapping organic radical spectra in OSC materials by demonstrating the application of relaxation-filtered EPR and EDNMRinduced EPR for direct separation of the two spectral components in the blend. The obtained magnetic resonance parameters of the ITIC radical will be compared to the results of density functional theory (DFT) calculations.

\section{Materials and methods}

\subsection{Sample preparation}

The organic semiconductors PBDB-T and ITIC were obtained from Solarmer Energy 
Inc. (El Monte, California, USA) and applied without further purification. Regioregular poly(3-hexylthiophene-2,5-diyl) (P3HT) (electronic grade, $99.995 \%$ pure) and PCBM (99\% pure) for reference measurements were purchased from Sigma-Aldrich (St. Louis, Missouri, USA) and Solenne (Groningen, Netherlands), respectively. The chemical structures of these compounds are given in Figure 1. Both blend and pure donor/acceptor films were drop-casted from chlorobenzene solutions. For all the BHJ blends, the solutions were prepared with a 1:1 donor:acceptor weight ratio, amounting to a total weight over solvent volume of $20 \mathrm{mg} / \mathrm{ml}$. For the pure component solutions, the same concentration of $20 \mathrm{mg} / \mathrm{ml}$ was used. EPR samples were prepared by scratching off the drop-cast film dried on a glass substrate and transferring the collected material into a W-band EPR quartz sample tube of appropriate size (I.D. $0.6 \mathrm{~mm}$, O.D. $0.84 \mathrm{~mm}$ ). The filling up to a few $\mathrm{mm}$ of the tube is not very dense hence no quantitative comparison of signal intensities in different samples can be made. Taking into account that the thickness of the drop-cast films is larger than the submicron penetration depth of the laser light, the illumination during experiments is expected to be inhomogeneous. In order to avoid photo-oxidation of the samples, the drop-cast films were dried in the dark under $\mathrm{N}_{2}$ atmosphere.

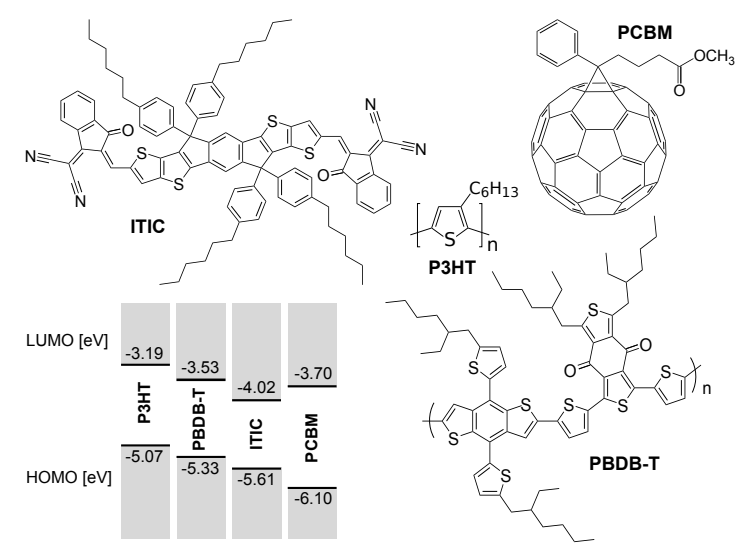

Figure 1: Chemical structure and band diagram of the materials under study. HOMO/LUMO molecular energy-levels were obtained from $[6,31]$.

\subsection{EPR experiments}

High-field EPR experiments were performed on a Bruker Elexsys E680 W-band EPR spectrometer (Bruker Biospin, Rheinstetten, Germany), equipped with a standard single-mode cylindrical resonator from Bruker and a continuous flow cryostat and superconducting magnet from Oxford Instruments (UK). To allow for light-induced experiments, the sample could be illuminated inside the cavity with the $532 \mathrm{~nm}$ output of a frequency-doubled continuous-wave Nd:YAG laser (CNI lasers (Changchun, China), MGL-III-532$300 \mathrm{~mW}$ ) via a fused silica optical fiber (core diameter: $600 \mu \mathrm{m}$ ) into the quartz sample tube acting as a light guide $(20 \mathrm{~mW}$ laser power at the end of the fiber). All experiments were performed at $20 \mathrm{~K}$ and with a shot repetition rate of $50 \mathrm{~Hz}$, unless stated otherwise. To avoid signal distortion due to saturation and fast-passage effects at low temperatures, the field-swept EPR spectra were recorded by electron spin echo (ESE) detection using the pulse sequence $\pi / 2-\tau-\pi-\tau-$ echo with $t_{\pi / 2}=128 \mathrm{~ns}, t_{\pi}=256 \mathrm{~ns}$ and interpulse delay $\tau=412$ ns. A two-step phase-cycle was applied to remove all unwanted echoes. All ESE-detected EPR spectra are presented as difference spectra (light-minus-dark) in order to determine the lightinduced components. The original dark spectra can be found in the SI. To allow for direct comparison, all of the presented spectra were brought to the same mw frequency $(94.02 \mathrm{GHz})$ to account for small frequency differences during the experiments.

(a)

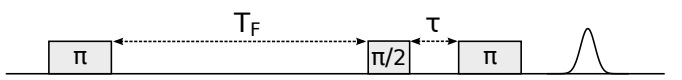

(b)

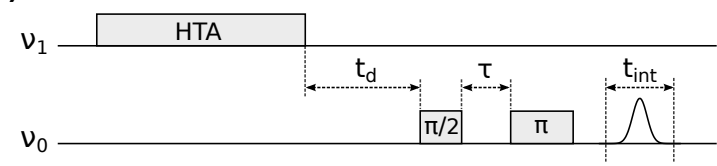

Figure 2: Pulse sequences used in this work: (a) relaxationfiltered EPR and (b) EDNMR with echo detection. Symbols are explained in the text.

In the field-swept relaxation-filtered EPR experiments, an additional $\pi$ inversion pulse $\left(t_{\pi}=\right.$ $256 \mathrm{~ns}$ ) was applied prior to the ESE detection sequence. The corresponding pulse sequence is shown in Figure 2(a) and is applied during illumination of the sample. The pulse lengths and interpulse delay of the detection sequence were the same as above. The procedure for optimizing the filter time $T_{\mathrm{F}}$ is described in detail in the results section. In all relaxation-filtered EPR experiments a four-step phase-cycle was applied to get rid of unwanted echoes.

ELDOR-detected NMR (EDNMR) was performed under illumination using the pulse sequence shown in Figure 2(b). The EDNMR spectrum is obtained by sweeping the high-turning angle (HTA) pulse 
frequency $\nu_{1}$ about the resonant mw frequency $\nu_{0}$ of the detection Hahn echo sequence at fixed magnetic field. The length of the HTA pulse was optimized to $24 \mu$ s by means of a nutation experiment where the intensity of the ${ }^{14} \mathrm{~N}$ EDNMR transition was recorded as a function of the HTA pulse length. The mw field amplitude of the HTA pulse is fixed in this set-up to $\omega_{1} / 2 \pi=2.0 \mathrm{MHz}$. Between the HTA pulse and the detection sequence a delay $t_{d}$ of $4 \mu \mathrm{s}$ was included. In the ESE detection sequence the same pulse lengths and interpulse delay as in the field-swept EPR experiments were applied. To ensure sufficient sensitivity, the echo integration window $t_{\text {int }}$ was set to $500 \mathrm{~ns}$, yielding a maximal resolution of $\sim 3 \mathrm{MHz}$ (according to the empirical EDNMR linewidth dependence described in [32]). The EDNMR-induced EPR experiments are described in detail in the results section. No phasecycle was used in the EDNMR experiments.

The simulations of the EPR spectra were performed with the Easyspin software package (version 5.1.3) [33].

\subsection{DFT calculations}

Spin-unrestricted DFT calculations of the negative ITIC radical were performed with the ORCA package (version 2.9.1) [34-36]. The structure geometry was first optimized using the BP86 functional [37] and the SVP basis set [38] for all atoms. Single-point computations of the principal $g$-values of the anion were performed using the B3LYP functional [37, 39] combined with the EPR-II basis set [40] for H, C, N, O and the TZV-PP basis set [38] for $\mathrm{S}$. In all calculations, the 4-hexylphenyl side groups were replaced by a methyl group to limit the computation time of the geometry relaxation step. Moreover, including the side groups did not significantly affect the magnetic resonance parameters (see SI). All three possible conformers of the molecular structure were taken into account. The effect of the surrounding medium could be incorporated in the calculations via the conductor-like screening model (COSMO) implemented in ORCA [41] assuming a dielectric constant $\epsilon=3.5$ typical for an organic semiconductor blend environment [42]. However, no pronounced effect of the dielectric medium on the optimized geometries and magnetic resonance parameters was found (see SI). Therefore all further calculations were performed assuming a vacuum.

\section{Results and discussion}

Charge transfer in materials for organic photovoltaics is driven by the energetic offset between both highest occupied molecular orbital (HOMO) and lowest unoccupied molecular orbital (LUMO) energy levels of the donor and acceptor materials. Regardless of whether the exciton is created by photon absorption in the donor or acceptor compound, the HOMO and LUMO offsets at the donor/acceptor interface will provide the necessary driving force to break up the coulombically bound exciton into two charge-separated states: a positive polaron on the (electron) donor and a negative polaron on the (electron) acceptor. The band diagram with the HOMO and LUMO molecular energies of the materials under study is shown in Figure 1 and demonstrates that charge transfer is possible in the blend combinations under study.

Figure 3 shows the light-induced ESE-detected EPR spectrum of the PBDB-T:ITIC blend and the pure donor and acceptor control samples. The corresponding $\mathrm{X}$-band light-induced continuous-wave EPR is included in the SI. Comparison with the pure samples confirms that the light-induced EPR in the blend stems from charge-transfer radicals. However, the two-component character of the spectrum could not be spectrally resolved, even at Wband frequency.

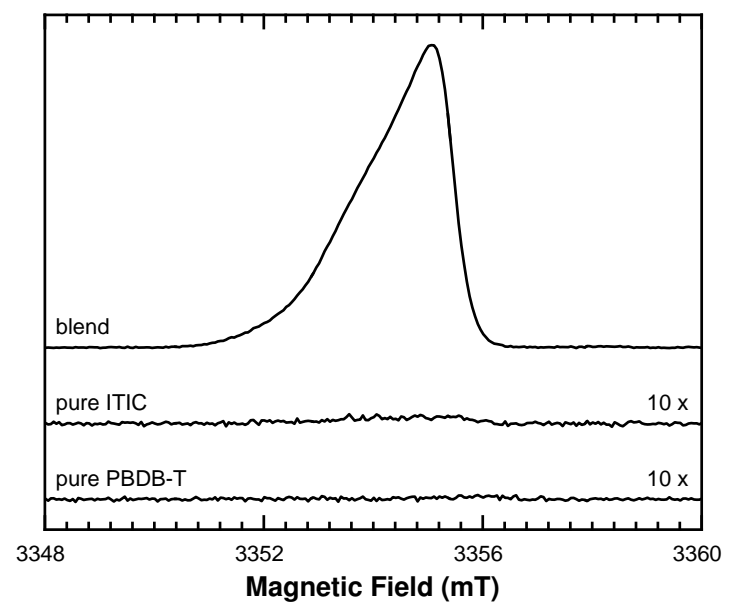

Figure 3: W-band light-induced ESE-detected EPR spectra of the 1:1 PBDB-T:ITIC blend and the pure donor and acceptor control samples. The latter are shown magnified (x10).

In a first approach to obtain non-overlapping spectra of the positive and negative radical we made new blends combining PBDB-T (ITIC) with a suitable acceptor (donor) compound with well characterized $\boldsymbol{g}$-tensor, known to be as much as possible outside the spectral region of interest. The spectra of the individual components resolved by blending with other compounds will later serve as a reference for the separation of the PBDB-T:ITIC blend spectrum by means of other pulsed techniques. The paradigm fullerene acceptor PCBM is an ideal 

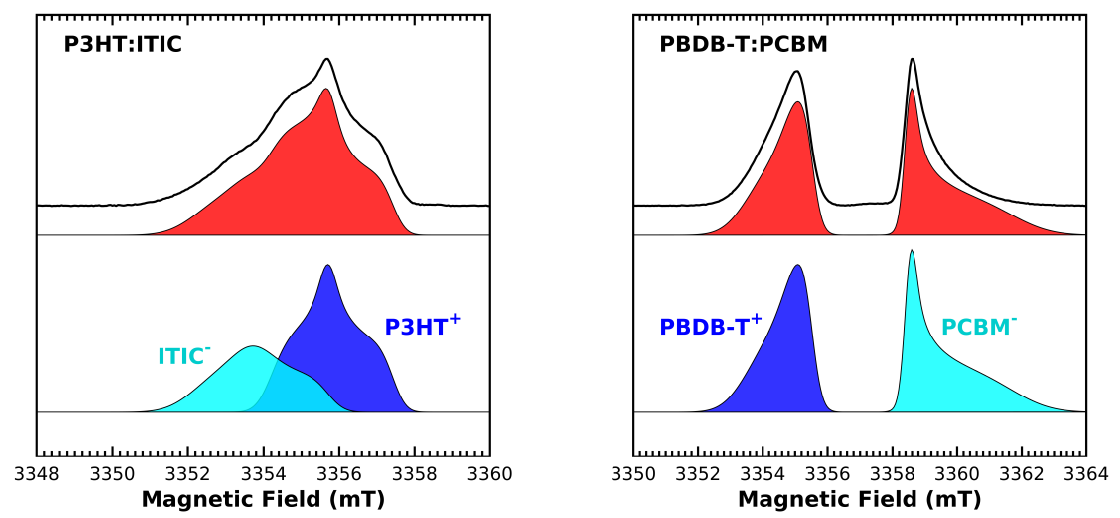

Figure 4: W-band light-induced EPR spectra of 1:1 P3HT:ITIC (left) and 1:1 PBDB-T:PCBM (right). Black: experimental spectra. Blue: simulation of the positive polaron $\mathrm{P}^{+}$on the polymer. Cyan: simulation of the negative polaron $\mathrm{P}^{-}$on the acceptor molecule. Red: sum of the two simulations. The principal $g$-values and linewidths used in the simulations are given in Table 1.

candidate to be combined with PBDB-T due to the nearly axial $\boldsymbol{g}$-tensor of the anion, resulting in a narrow high-field line close to $g \approx 2.0000$, expected to be well separated from the one of the polymer polaron $[22,43]$. In order to resolve the ITIC radical spectrum, we opted for a blend with the wellstudied polymer P3HT for which the principal values of the polaron $\boldsymbol{g}$-tensor have been determined to be $g_{x}=2.0009, g_{y}=2.0019, g_{z}=2.0028[7,8]$. Successful charge transfer in P3HT:ITIC blends has already been established by Qin et al. [44], yet solar cells based on this donor-acceptor combination exhibited rather poor PCEs of $1.25 \%$.

Table 1: Principal components of the $\boldsymbol{g}$-tensors of the positive polaron on the polymers and the negative acceptor radicals. The experimental $g$-values and linewidths $\Delta B$ were determined by simulation of the W-band EPR data shown in Figure 4 and 5. The estimated error in the experimental $g$-values and linewidths is \pm 0.0001 and $\pm 0.04 \mathrm{mT}$, respectively. Because of spectral overlap with the polymer polaron in both blends, a slightly larger uncertainty of \pm 0.0003 is assumed for the $g_{x}$ value of the ITIC anion.

\begin{tabular}{|c|ccc|c|}
\hline & $g_{x}$ & $g_{y}$ & $g_{z}$ & $\Delta B(\mathrm{mT})$ \\
\hline PBDB-T $^{+}$ & 2.0019 & 2.0022 & 2.0032 & $(0.50,0.89,1.43)$ \\
ITIC $^{-(a)}$ & 2.0022 & 2.0034 & 2.0044 & $(0.79,1.00,1.43)$ \\
ITIC $^{-(b)}$ & 2.0025 & 2.0034 & 2.0044 & $(0.79,1.00,1.43)$ \\
P3HT $^{+}$ & 2.0011 & 2.0022 & 2.0031 & $(0.64,0.41,0.79)$ \\
PCBM $^{-}$ & 1.9981 & 2.0000 & 2.0002 & $(2.14,0.32,0.32)$ \\
\hline
\end{tabular}

(a) obtained from P3HT:ITIC blend (by fitting the spectrum in Figure 4).

(b) obtained from PBDB-T:ITIC blend (by fitting the spectrum in Figure 5).

The light-induced EPR spectra of the PBDBT:PCBM and P3HT:ITIC blends shown in Figure 4 demonstrate the efficient generation of charge-separated states under illumination in both BHJ systems. For the PBDB-T:PCBM blend a complete separation of the positive and negative radical spectra is obtained. As could be expected

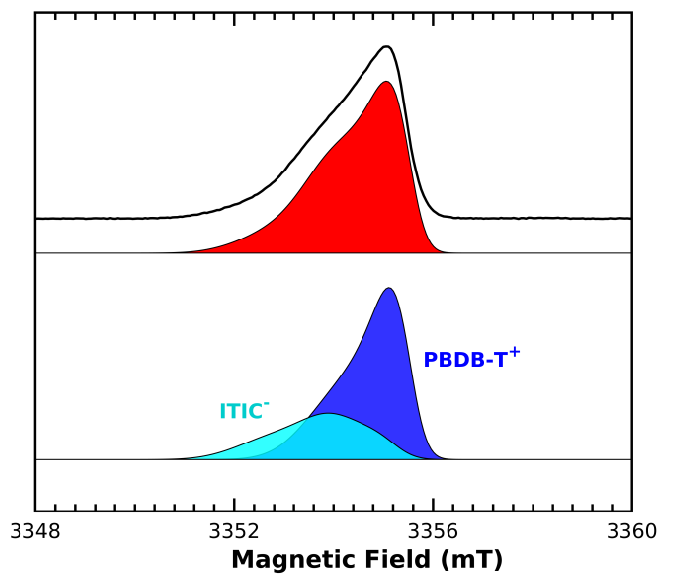

Figure 5: Simulation of the W-band light-induced EPR spectrum of the 1:1 PBDB-T:ITIC blend. The color coding is the same as in Figure 4. The simulations were performed by optimizing the principal $g$-values obtained from fitting the spectra in Figure 4 which can be found in Table 1. All optimized parameters were the same within the experimental error, except for the $g_{x}$ value of the ITIC anion, which was taken slightly larger $\left(\Delta g_{x}=0.0003\right)$ to accurately reproduce the experimental spectrum.

from the HOMO/LUMO levels in Figure 1, PCBM acts as an acceptor in combination with PBDB$\mathrm{T}$, producing the well-known PCBM anion spectrum at the high-field side $[22,43]$. Hence the low-field spectrum can be unambiguously assigned to the positive PBDB-T polaron. In the case of P3HT:ITIC, the EPR spectra of the individual components partially overlap. However, comparison of the spectrum with the available literature on effective $g$-values and typical W-band linewidths of the positive P3HT polaron [7] allows for identification of this polaron on the high-field side of the overlapping spectrum. Hence the donor character of P3HT in combination with ITIC is confirmed and simultaneous fitting of the blend spectrum en- 

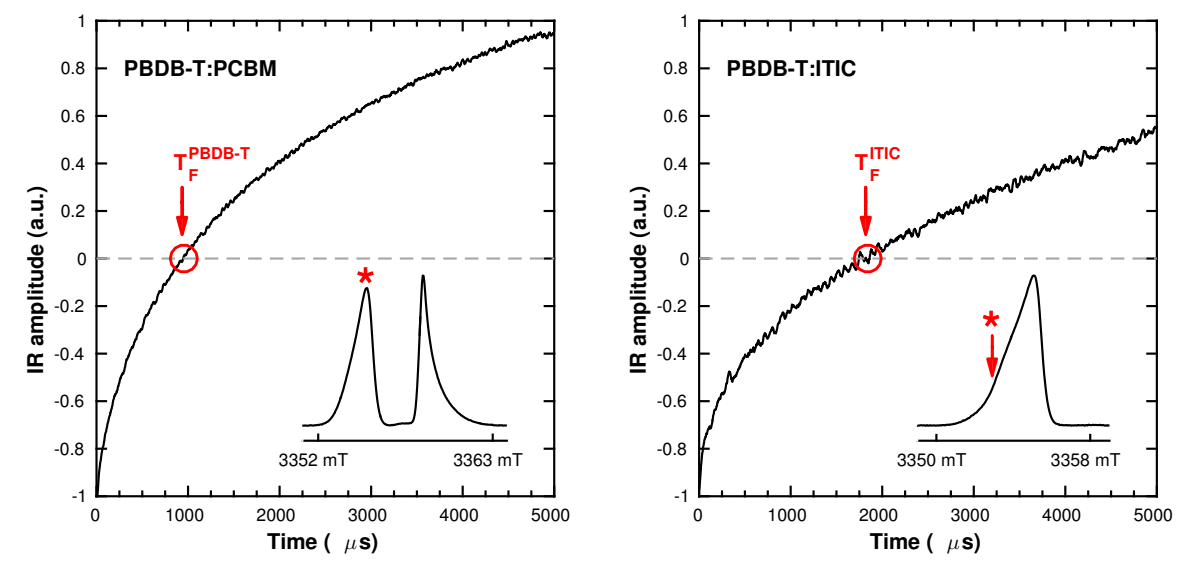

Figure 6: W-band inversion-recovery time traces of the PBDB-T polaron in the PBDB-T:PCBM blend (left) and the ITIC anion in the PBDB-T:ITIC blend (right). In both panels, the magnetic field position at which the time trace is recorded is indicated by a red asterisk in the corresponding EPR spectrum shown in the inset.

ables extraction of the $\boldsymbol{g}$-tensor components of the ITIC negative radical. The principal values of the $\boldsymbol{g}$-tensors of the charge-transfer radicals obtained by simulation of the experimental spectra are summarized in Table 1.

\subsection{Relaxation-filtered EPR}

The above spectral separation approach, although successful for the current case, is not generic. It depends on the condition that suitable other blend partners can be found that allow this spectral separation and on the pre-assumption that the nature of the positive or negative polaron is not affected by the blend partner. Therefore, two other methods are tested to separate the overlapping EPR spectrum in the PBDB-T:ITIC blend. First we will disentangle the overlapping spectra by means of the relaxation-filtering technique that relies on the difference in longitudinal relaxation times $T_{1}$ of the spectral constituents. The different relaxation properties of the two radicals become evident when recording the ESE-detected EPR spectrum with varying shot repetition times (SRT) (see SI). As shown in Figure SI.3 the low-field part of the spectrum clearly reduces when the SRT is lowered from $20 \mu$ s to $8 \mu$ s, suggesting that this part of the spectrum belongs to a slower relaxing species. Such differences in $T_{1}$ times can be exploited in a relaxation-filtered EPR experiment (sequence in Figure 2(b)). The actual relaxation filtering was achieved by introducing an inversion $\pi$-pulse prior to the ESE detection sequence when recording the field-swept spectrum. The filter time $T_{\mathrm{F}}$ between the inversion-pulse and the first detection pulse is chosen such that it matches the zero-crossing of the inverted magnetization of an individual component in a classic inversion-recovery (IR) exper- iment. Hence, the resulting inversion-recovery filter (IRf) will selectively suppress the corresponding component in the recorded echo and its contribution to the EPR spectrum will vanish. Clearly, the success of the relaxation-filtering technique depends on an accurate determination of the filter times of the overlapping components.

Determining $T_{\mathrm{F}}$ from the zero-crossing in an IR experiment requires that no other contributions from overlapping components are present at the field position at which the time-trace is recorded. In principle, one could extract the filter times of a multi-component IR trace by fitting the magnetization decay with a set of different $T_{1}$ parameters. However, often the time trace is ill-described by a single exponential decay and multiple parameters are required to fit the magnetization decay of a single component [24]. As such, the increased parameter space necessary for fitting each paramagnetic center greatly reduces the accuracy of relaxation times determined from a multi-component fit, particularly for similar $T_{1}$ times, as expected for a mixture of organic radicals. In case of completely overlapping spectra that do not exhibit isolated single-component contributions, a possible approach is to record the IR time traces in a 2dimensional experiment wherein the magnetic field is swept in the second dimension, as demonstrated in [26]. An inverse Laplace transformation of the spectrum along the time dimension then allows to separate the individual components according to their respective relaxation rates.

For the PBDB-T:ITIC blend, the $T_{\mathrm{F}}$ of the ITIC radical could be obtained directly by performing an IR experiment at the low-field side of the spectrum, where no contribution from the polymer radical is present, as follows from the ESE-detected EPR 

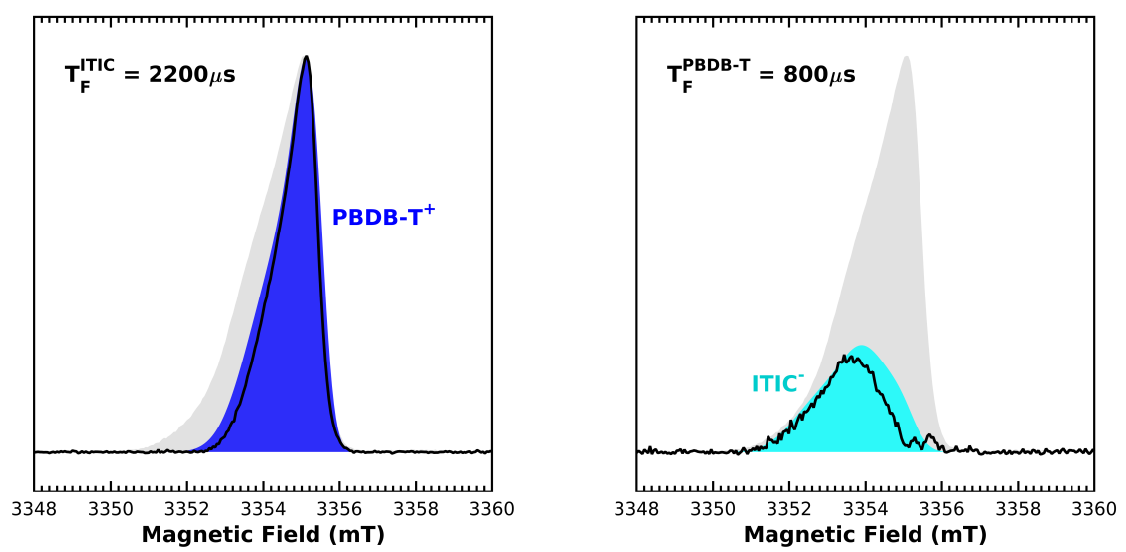

Figure 7: W-band IRf field-swept EPR spectra of the PBDB-T:ITIC blend. Left: IRf field-swept spectrum with $T_{\mathrm{F}}=T_{\mathrm{F}}^{\mathrm{ITIC}}$, i.e. with the ITIC anion contribution filtered out. Right: IRf field-swept spectrum with $T_{\mathrm{F}}=T_{\mathrm{F}}^{\mathrm{PBDB}-\mathrm{T}}$, i.e. with the PBDB$\mathrm{T}$ polaron contribution filtered out. For reference purposes, the full ESE-detected PBDB-T:ITIC spectrum is displayed in the background in gray. The simulations of the PBDB-T polaron (blue) and the ITIC anion (cyan) obtained from fitting the EPR spectrum in Figure 5 are shown for comparison.

with different repetition rates and confirmed by the P3HT:ITIC experiments. On the other hand, the EPR spectrum of the positive polaron in PBDB-T completely overlaps with the ITIC anion contribution. Therefore we recorded the IR time trace of the polymer polaron in the PBDB-T:PCBM blend where its EPR spectrum is fully resolved. Both IR time traces are shown in Figure 6. From the zero-crossing of the magnetization decays in Figure $6, T_{\mathrm{F}}=1850 \mu \mathrm{s}$ is obtained for the ITIC radical and $T_{\mathrm{F}}=950 \mu$ s for the PBDB-T radical. The different environment of the PBDB-T polaron in the two blends can be expected to slightly affect the $T_{1}$ relaxation time. Therefore a further optimization of the $T_{\mathrm{F}}$ of the PBDB-T component was performed by means of a 2-dimensional IRf experiment in the PBDB-T:ITIC blend where the time axis was limited to a $T_{\mathrm{F}}$ range centered about $T_{\mathrm{F}}=950 \mu \mathrm{s}$ (not shown). Since the optimal $T_{\mathrm{F}}$ corresponds to the zero-crossing of the echo contribution of the PBDB-T polaron, it can be accurately determined from the $2 \mathrm{D}$ experiment via the sign change of the PBDB-T residuals in the fieldswept spectra upon varying $T_{\mathrm{F}}$. Also for the ITIC radical the same procedure was applied to further optimize the $T_{\mathrm{F}}$.

Figure 7 shows the W-band IRf field-swept spectra of the charge-transfer radicals in the PBDBT:ITIC blend. Optimization of the filtering times to obtain complete suppression of a single component in the EPR spectrum resulted in slightly different $T_{\mathrm{F}}$ values - $800 \mu$ s and $2200 \mu \mathrm{s}$ for PBDB-T ${ }^{+}$ and ITIC $^{-}$respectively - than those obtained from the IR experiments shown in Figure 6. For both radicals a successful IR filtering was achieved, resulting in nearly $100 \%$ suppression of a single com- ponent. The separated PBDB-T polaron spectrum obtained by IR filtering in the PBDB-T:ITIC blend is in excellent agreement with the resolved singlecomponent spectrum obtained by blending PBDB$\mathrm{T}$ with PCBM (presented in Figure 4). For the ITIC anion, however, the IRf spectrum exhibits significantly smaller $\boldsymbol{g}$-anisotropy in comparison to the ESE-detected P3HT:ITIC spectrum. The range $3354-3356 \mathrm{mT}$ where the contribution of the ITIC anion has been suppressed in the IRf field-sweep, corresponds to the region where the two components strongly overlap. Moreover, in the regular ESE-detected spectrum, the intensity of the PBDB-T signal in this region is roughly four times larger than that of the ITIC radical contribution. Hence, the effectivity of the IRf technique seems to diminish significantly when the larger PBDB-T component is suppressed in the combined echo, thereby canceling out the highfield ITIC anion spectrum. A key factor contributing to a distortion of IRf spectra, is an orientational dependence of the $T_{1}$ relaxation time of one of the components. In that case, the corresponding $T_{\mathrm{F}}$ cannot be unambiguously determined and complete suppression of the component over its entire field range is impossible. The relaxationfiltered EPR technique therefore can be at its limits when one of the components displays significant $T_{1}$ anisotropy, especially when this component has higher ESE signal intensity in the strongly overlapping regions, or when differences in $T_{1}$ relaxation rates are small. However, for neither the PBDB$\mathrm{T}$ nor the ITIC radical, significant $T_{1}$ anisotropies were observed, yet, the anomalous features in the high-field region of the filtered ITIC anion spectrum seem to indicate weak $T_{1}$ anisotropy for the PBDB-T polaron. A more detailed study of $T_{1}$ 

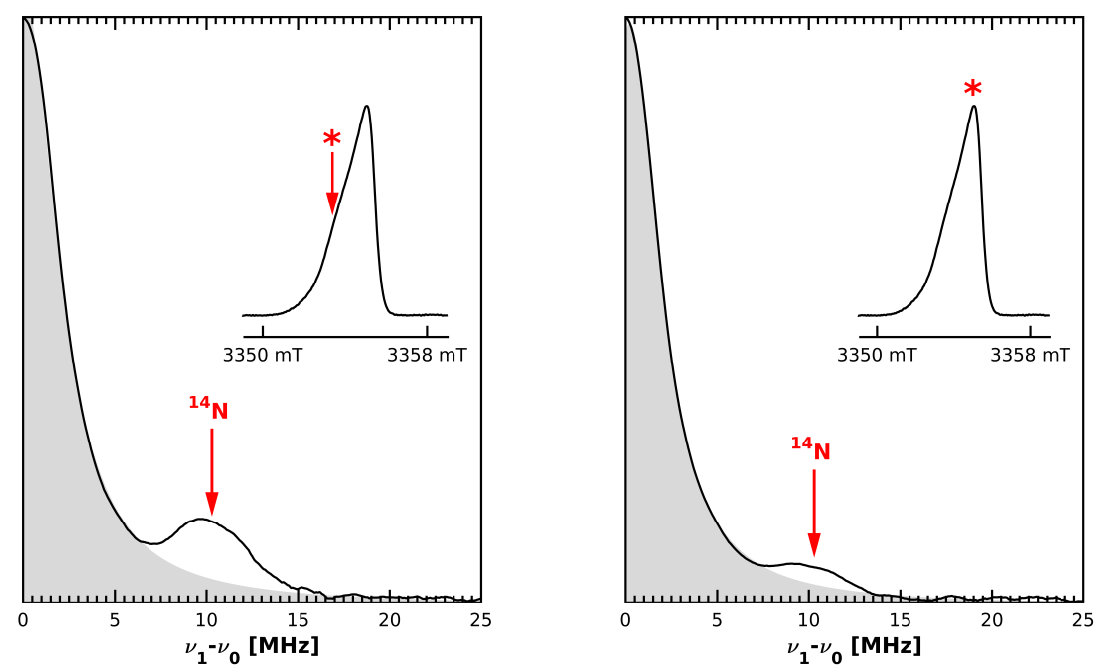

Figure 8: W-band EDNMR spectrum of PBDB-T:ITIC recorded at $B=3353.6 \mathrm{mT}$ (left) and $B=3355.2 \mathrm{mT}$ (right). A fit of the central Lorentzian lineshape at $\Delta \nu=0$ is shown in gray. In both panels, the magnetic field position at which the EDNMR spectrum is recorded is indicated by a red asterisk in the EPR spectrum in the inset. Since the spectra are symmetrical, only the right-hand side is shown.

anisotropy of the PBDB-T polaron can be found in the SI. The validity of the IRf method can easily be checked since combination of the two obtained spectra should lead to the initial EPR spectrum of the blend (Figure SI.5).

\section{2. $E D N M R$-induced $E P R$}

An alternative approach to access the acceptor radical spectrum directly from the PBDB-T:ITIC blend can be devised by exploiting the presence of weakly coupled ${ }^{14} \mathrm{~N}$ nuclei in the ITIC molecule, similar to the electron nuclear double resonance (ENDOR) induced EPR technique introduced by Hyde [45]. At W-band frequencies, however, ENDOR has a significantly smaller sensitivity for low$\gamma$ nuclei than EDNMR [28]. Recently Nalepa et al. [32] compared the signal-to-noise ratios of the ENDOR and EDNMR spectra of the ${ }^{14} \mathrm{~N}$ hyperfine coupling in a nitroxide radical. They found that the sensitivity of EDNMR is superior to ENDOR by a factor of 30 , which allows for considerably shorter recording times. Hence we opted for EDNMR to detect the ${ }^{14} \mathrm{~N}$ coupling of the ITIC radical and implemented EDNMR-induced EPR experiments, analogously to the ENDOR-based technique demonstrated by Hyde.

Figure 8 presents the EDNMR spectra of PBDBT:ITIC recorded at two different field positions to assess the g-anisotropy of the ITIC anion established earlier by fitting the P3HT:ITIC spectrum shown in Figure 4. Because of the weak hyperfine coupling of the unpaired electron with the $4 \mathrm{~N}$ nuclei, all of the three allowed EPR transitions of the $m_{I}$ multiplet fall within the detection pulse bandwidth. Hence the obtained EDNMR spectrum is symmetrical, apart from possible minor deviations due the position of the observer $\mathrm{mw}$ frequency in the non-symmetrical resonator bandwidth. Therefore only the right-hand side of both EDNMR spectra is shown in Figure 8. At both field positions, the EDNMR spectrum exhibits a broad peak centered about $\Delta \nu=10 \mathrm{MHz}$, corresponding to the Larmor frequency of ${ }^{14} \mathrm{~N}$. For weakly coupled nuclei, the splitting of the EDNMR peaks about the Larmor frequency is determined by the strength of the hyperfine coupling and nuclear quadrupole coupling [46]. In this case, however, the hyperfine and quadrupole couplings are small and not resolved. Double quantum transitions (with $\Delta m_{I}= \pm 2$ ) could be expected to appear centered around $20 \mathrm{MHz}$, i.e. twice the Larmor frequency of ${ }^{14} \mathrm{~N}$.

The observation of ${ }^{14} \mathrm{~N}$ hyperfine couplings in the EDNMR spectrum at the high-field side of the PBDB-T:ITIC spectrum confirms the considerable $\boldsymbol{g}$-anisotropy of the ITIC anion obtained earlier from the simulation of the P3HT:ITIC blend (Figure 4). As a next step, the ${ }^{14} \mathrm{~N}$ hyperfine couplings can be used to directly isolate the ITIC anion contribution to the overlapping spectrum of the blend by means of EDNMR-induced EPR. The separate spectrum of a single component can be obtained by recording the EPR spectrum using the EDNMR sequence with the mw offset $(\Delta \nu)$ fixed, once on resonance, once off resonance with a ${ }^{14} \mathrm{~N}$ nuclear frequency, and then taking the difference of the two traces. Hence, when the nuclear contri- 
bution in the EDNMR spectrum can be solely ascribed to one paramagnetic center, the corresponding EDNMR-induced EPR spectrum will reflect the EPR spectrum of this center. We remark that this approach is valid here because of the weak dependence of the EDNMR signal on the orientational selection which ensures that the depth of the hole burnt by the HTA pulse can be assumed proportional to the ITIC anion EPR signal in its entire field range. Figure 9 shows the EDNMR-induced EPR spectrum obtained for the ITIC radical by fixing the HTA frequency offset on / off resonance with the ${ }^{14} \mathrm{~N}$ hyperfine couplings.

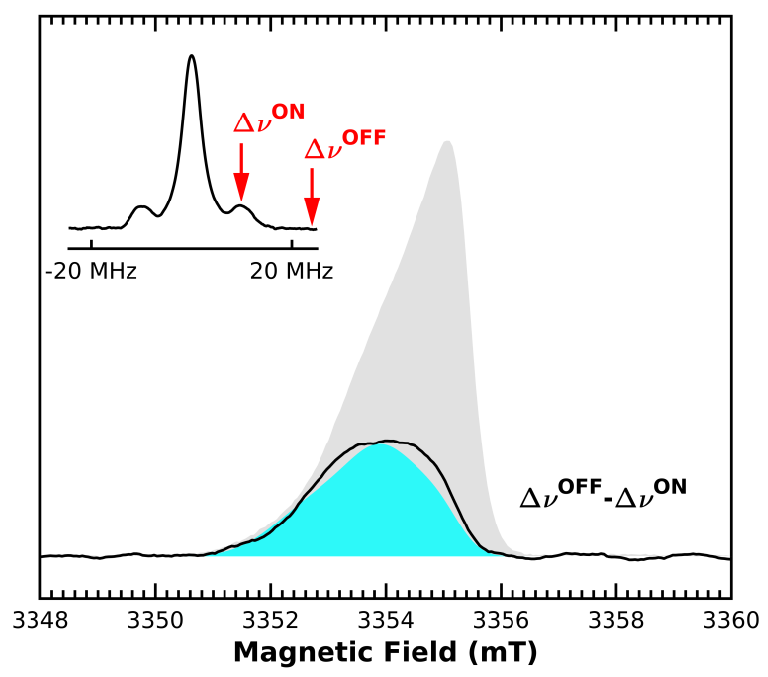

Figure 9: W-band EDNMR-induced EPR spectrum of PBDB-T:ITIC. This spectrum is obtained by subtracting the field-swept spectra recorded with HTA pulse frequency offsets $\Delta \nu^{\mathrm{OFF}}=25 \mathrm{MHz}$ and $\Delta \nu^{\mathrm{ON}}=10 \mathrm{MHz}$. The position of the mw frequency offsets in the $B=3353.6 \mathrm{mT}$ EDNMR spectrum are shown in the inset. For reference purposes, the ESE-detected PBDB-T:ITIC spectrum is displayed in the background in gray. The simulation of the ITIC anion (cyan) obtained from simultaneous fitting of the two radicals in the PBDB-T:ITIC blend spectrum in Figure 5 is shown for comparison.

The EDNMR-induced EPR spectrum of the ITIC anion is in good agreement with the simulation of the resolved spectrum obtained for the blend with P3HT. This result demonstrates the feasibility of EDNMR-induced EPR as a tool for separating overlapping EPR spectra. However, an important limitation of the technique is the presence of the long tails of the central Lorentzian that could overlap with the EDNMR transitions of low- $\gamma$ nuclei (see Figure 8). As such the EDNMR-induced spectrum will be superimposed with a residual contribution of the total spectrum and no perfect separation of the individual components can be achieved. This problem can be avoided to some extent by reducing the HTA pulse amplitude which is directly proportional to the width of the central Lorentzian line [28, 29]. Alternatively, one could perform a 2-dimensional EDNMR experiment where full EDNMR traces are recorded as a function of the magnetic field. In that case, the central Lorentzian can be subtracted in all of the EDNMR traces before performing the subtraction of a $\Delta \nu^{\mathrm{ON}}$ and a $\Delta \nu^{\mathrm{OFF}}$ field-swept spectra to obtain the EDNMRinduced EPR signal. This approach ensures that all residual contributions of the Lorentzian tails will be removed from the EDNMR-induced spectrum. In the case of PBDB-T:ITIC, no significant contribution of the Lorentzian tails in the form of PBDB-T polaron residuals is present in the EDNMR-induced EPR spectrum, even though the on-resonance trace was detected at a mw frequency offset of only $10 \mathrm{MHz}$.

For the narrow field sweeps of $12 \mathrm{mT}$ applied here, the field-dependence of the ${ }^{14} \mathrm{~N}$ Larmor frequency, which determines $\Delta \nu^{\mathrm{ON}}$ in this system, could safely be neglected. However, for broad field ranges, $\Delta \nu^{\mathrm{ON}}$ should in principle follow the field variation during the sweep, a requirement also encountered in ENDOR-induced EPR. This can be circumvented by recording a 2-dimensional EDNMR spectrum and integrating the relevant signal in the EDNMR traces over a sufficiently large window. The same approach is also applied in ENDOR-induced EPR, as illustrated by Kusakovskij et al. in [47] where the powder EPR spectrum of a single species in a mixture of sucrose radicals is reconstructed from its isolated ${ }^{1} \mathrm{H}$ hyperfine interaction in the ENDOR spectra. In a similar fashion, also orientationally dependent broadenings or shifts of an EDNMR signal due to strongly anisotropic hyperfine or quadrupolar couplings can be taken into account.

\subsection{DFT and comparison with experiment}

DFT computations were performed on a negatively charged ITIC radical to underpin the magnetic resonance parameters obtained experimentally. This singly charged radical state corresponds to the oxidation state of the ITIC acceptor directly after charge transfer with the PBDB-T polymer. In all calculations, the 4-hexylphenyl side groups of the ITIC molecule were replaced by a methyl group to limit the computation time of the geometry relaxation step. This approximation is justified because structural optimization of the molecular backbone should result in a planar structure, regardless of the side chain orientation and the spin density of the negative radical is expected to be delocalized along the conjugated $\pi$-system without leakage onto the 4-hexylphenyl side groups. To demonstrate that this truncation of the ITIC molecule 
did not affect the computed magnetic resonance parameters, we include a single $\boldsymbol{g}$-tensor calculation for the full structure in the SI for comparison. As shown in Figure 10, different conformers of the ITIC backbone exist depending on the cis/trans orientation of the side groups defined by two dihedral angles. All calculations were repeated for the three conformers.

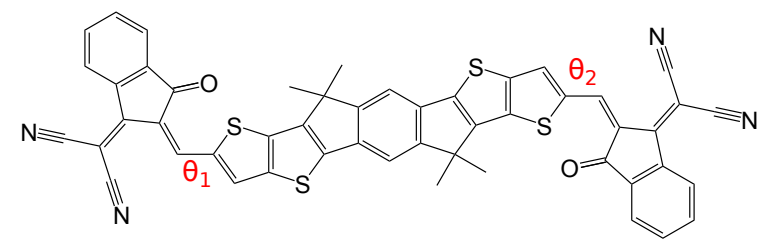

Figure 10: Different conformers of the ITIC backbone are possible depending on the dihedral angles $\theta_{1}$ and $\theta_{2}$.

As expected for a conjugated $\pi$-system, geometry optimization of all conformers resulted in planar structures. Table 2 summarizes the principal $\boldsymbol{g}$ tensor components obtained for each of the conformers and their total energy difference with respect to the ground state configuration. Similar energies were obtained for all of the conformers, suggesting that the EPR sample will contain a mixture of all possible ITIC configurations. However, since the calculated $g$-values are nearly identical for all conformers, their superposition is not expected to lead to significant $\boldsymbol{g}$-strain broadening of the experimental spectrum. Overall, the computed $\boldsymbol{g}$ anisotropy and isotropic $g$ values are slightly overestimated, but deviations are $\leq 0.001$. The $g_{y}$-value, which is oriented perpendicular to the molecular plane, seems to be the only $g$-value sensitive to the orientation of the $\theta_{1}$ and $\theta_{2}$ dihedral angles at this level of accuracy of the calculation. The agreement of the calculated $g_{x}$ and $g_{y}$ components with the experimental $g$-values is very good. The $g_{z}$ component, which lies within the plane of the $\pi$-system, perpendicular to the molecular backbone, is overestimated in all conformers.

Table 2: Comparison of the experimental and DFTcomputed principal $g$-values of the anion state of the truncated ITIC molecule. The three conformers of the ITIC backbone considered for the DFT calculations are defined in Figure 10. The total energy difference between the isomers is given in the last column. The experimental $g$-values were determined by simulation of the W-band EPR data shown in Figure 5.

\begin{tabular}{|c|ccc|c|}
\hline$\theta_{1}-\theta_{2}$ & $g_{x}$ & $g_{y}$ & $g_{z}$ & $\Delta E_{\text {tot }}(\mathrm{meV})$ \\
\hline $0-0$ & 2.0025 & 2.0036 & 2.0054 & 0 \\
$\pi-0$ & 2.0025 & 2.0038 & 2.0054 & +28.4 \\
$\pi-\pi$ & 2.0025 & 2.0039 & 2.0054 & +56.2 \\
\hline $\operatorname{Exp}$ & 2.0025 & 2.0034 & 2.0044 & \\
\hline
\end{tabular}

Figure 11 shows a contour plot of the spin-density of the ground state conformer of the anion state of the truncated ITIC molecule. As expected, the spin density is delocalized over the entire $\pi$-system of the molecule.

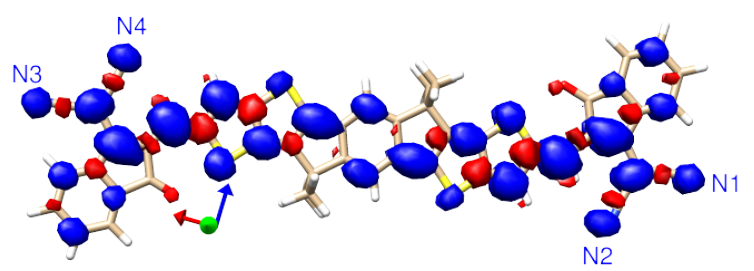

Figure 11: Spin density distribution of the negative polaron on the lowest energy conformer based on DFT calculations. Red: Negative spin density. Blue: Positive spin density. Contour levels were fixed at -0.0005 and 0.0005 , respectively. Principal axes $\boldsymbol{g}$-tensor: x (red), y (green), z (blue).

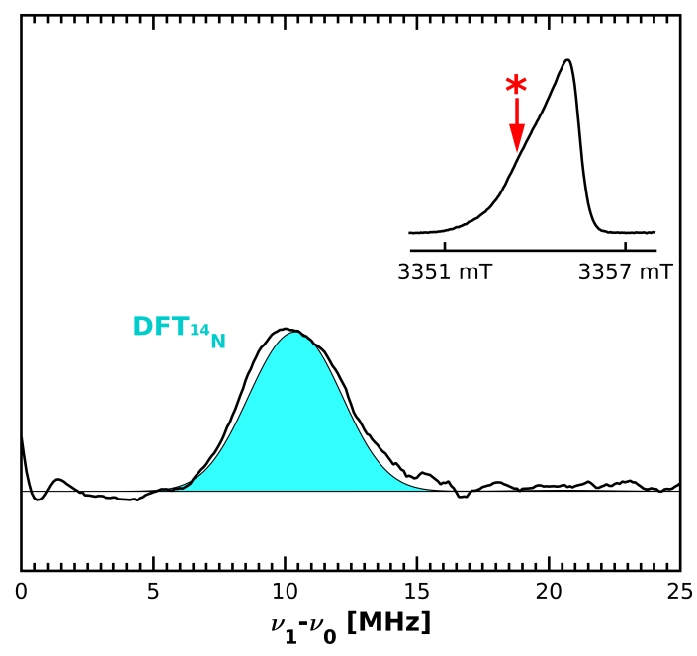

Figure 12: Simulation of the W-band EDNMR spectrum of PBDB-T:ITIC recorded at magnetic field $B=3353.6 \mathrm{mT}$ corresponding to the maximum EPR signal of the ITIC polaron. The central Lorentzian has been subtracted from the experimental spectrum (black). The simulation (cyan) is based on the DFT-computed ${ }^{14} \mathrm{~N}$ hyperfine and quadrupole tensors shown in Table 3 . A linewidth of $3 \mathrm{MHz}$ (full width at half maximum) was used for the simulation. The magnetic field position at which the EDNMR spectrum was recorded, is indicated by a red asterisk in the EPR spectrum in the inset.

The calculated ${ }^{14} \mathrm{~N}$ hyperfine and quadrupole tensors of the ground state conformer are given in Table 3. Since the molecular structure of the 0 0 conformer is symmetrical, also two-by-two symmetrical hyperfine and quadrupole couplings are obtained for the four nitrogen atoms in the system. Similar results were obtained for the other conformers (see SI). The DFT-computed hyperfine couplings allow for a simulation of the EDNMR spectrum of the ITIC radical, as shown in Figure 12. A linewidth of $3 \mathrm{MHz}$ (full width at half maximum) was assumed for the simulation accord- 
Table 3: DFT-computed ${ }^{14} \mathrm{~N}$ hyperfine and quadrupole tensors of the lowest energy conformer of the truncated ITIC radical.

\begin{tabular}{|c|c|c|c|c|c|c|c|}
\hline ITIC $^{-}$ & $A_{x}[\mathrm{MHz}]$ & $A_{y}[\mathrm{MHz}]$ & $A_{z}[\mathrm{MHz}]$ & $\alpha, \beta, \gamma\left[{ }^{\circ}\right]$ & $e^{2} q Q[\mathrm{MHz}]$ & $\eta[\mathrm{MHz}]$ & $\alpha, \beta, \gamma\left[{ }^{\circ}\right]$ \\
\hline $\mathrm{N}^{1}$ & -0.27 & 3.46 & -0.58 & $0,19,90$ & -2.98 & 0.44 & $0,19,90$ \\
$\mathrm{~N}^{2}$ & -0.65 & 3.61 & -0.31 & $-3,2,-88$ & -3.02 & 0.40 & $-4,0,-87$ \\
$\mathrm{~N}^{3}$ & -0.58 & -0.28 & 3.46 & $-19,90,0$ & -2.98 & 0.44 & $-20,90,0$ \\
$\mathrm{~N}^{4}$ & -0.31 & -0.65 & 3.62 & $2,90,0$ & -3.02 & 0.40 & $-8,0,-82$ \\
\hline
\end{tabular}

ing to an estimate of the EDNMR spectral resolution based on the empirical relation described in [32]. Clearly, the width of the ${ }^{14} \mathrm{~N}$ feature in the EDNMR spectrum is in excellent agreement with the DFT-computed hyperfine and quadrupole couplings and corroborates its assignment.

\section{Conclusion}

In this work we present a full characterization of the $\boldsymbol{g}$-tensor of the light-induced polarons in the fullerene-free OSC blend PBDB-T:ITIC. Different approaches for disentangling the overlapping organic radical spectra were tested. For OSC materials, combining the donor and acceptor with other blend partners is a viable method for obtaining fully-resolved spectra. This method is, however, not generic and direct spectral separation techniques such as relaxation-filtered EPR and EDNMR-induced EPR are required when no suitable blend partners are available. Despite the small $T_{1}$ difference, relaxation-filtered EPR was highly succesful to reproduce the spectrum of the PBDB-T polaron. It featured, however, a significant distortion of the weaker spectrum of the ITIC radical in the magnetic field region where the two contributions were strongly overlapping. Yet, the main advantage of this technique is its general applicability to any mixture of paramagnetic species, given the $T_{1}$ relaxation times can be assumed isotropic. In contrast, EDNMR-induced EPR relies on the presence of a unique magnetic nucleus in one of the overlapping components, hence it is less widely applicable. However, when a distinctive EDNMR signal is available, this method is not limited by requirements such as incomplete overlap between the EPR spectra, sufficient difference in $T_{1}$ relaxation times or absence of anisotropy in the relaxation properties which must be met for the $T_{1}$ based IRf method. In the case of the PBDBT:ITIC blend, the EDNMR-induced EPR method allowed for a good recording of the EPR contribution of the ITIC polaron based on its hyperfine coupling to surrounding ${ }^{14} \mathrm{~N}$ nuclei which are absent in the polymer. We consider the methods demonstrated in this work on the PBDB-T:ITIC blend to be broadly applicable for the unambiguous characterization of the $\boldsymbol{g}$-tensor of OSC materials in case of strongly overlapping EPR spectra.

\section{Acknowledgements}

The authors want to acknowledge the Research Foundation Flanders (FWO - Vlaanderen) for support of this work through the project G0B6715N and the PhD fellowship of M. Van Landeghem.

\section{References}

[1] Y. Lin, X. Zhan, Non-fullerene acceptors for organic photovoltaics: an emerging horizon, Mater. Horizons 1 (5) (2014) 470. doi:10.1039/C4MH00042K.

[2] A. F. Eftaiha, J.-P. Sun, I. G. Hill, G. C. Welch, Recent advances of non-fullerene, small molecular acceptors for solution processed bulk heterojunction solar cells, J. Mater. Chem. A 2 (5) (2014) 1201-1213. doi:10.1039/C3TA14236A.

[3] S. Li, W. Liu, C.-Z. Li, M. Shi, H. Chen, Efficient Organic Solar Cells with Non-Fullerene Acceptors, Small 13 (37) (2017) 1701120. doi:10.1002/smll.201701120.

[4] J. Zhao, Y. Li, G. Yang, K. Jiang, H. Lin, H. Ade, W. Ma, H. Yan, Efficient organic solar cells processed from hydrocarbon solvents, Nat. Energy 1 (2) (2016) 15027. doi:10.1038/nenergy.2015.27.

[5] W. Zhao, D. Qian, S. Zhang, S. Li, O. Inganas, F. Gao, J. Hou, Fullerene-Free Polymer Solar Cells with over $11 \%$ Efficiency and Excellent Thermal Stability, Adv. Mater. 28 (23) (2016) 4734-4739. doi:10.1002/adma.201600281.

[6] S. Li, L. Ye, W. Zhao, S. Zhang, S. Mukherjee, H. Ade, J. Hou, Energy-Level Modulation of Small-Molecule Electron Acceptors to Achieve over 12\% Efficiency in Polymer Solar Cells, Adv. Mater. 28 (42) (2016) 94239429. doi:10.1002/adma.201602776.

[7] A. Aguirre, P. Gast, S. Orlinskii, I. Akimoto, E. J. J. Groenen, H. El-Mkami, E. Goovaerts, S. Van Doorslaer, Multifrequency EPR analysis of the positive polaron in $\mathrm{I}_{2}$-doped poly(3-hexylthiophene) and in poly[2-methoxy-5-(3,7-dimethyloctyloxy)]-1,4phenylenevinylene, Phys. Chem. Chem. Phys. 10 (47) (2008) 7129-7138. doi:10.1039/b811419f.

[8] J. Niklas, K. L. Mardis, B. P. Banks, G. M. Grooms, A. Sperlich, V. Dyakonov, S. Beaupré, M. Leclerc, T. Xu, L. Yu, O. G. Poluektov, HighlyEfficient Charge Separation and Polaron Delocalization in Polymer-Fullerene Bulk-Heterojunctions: A Comparative Multi-Frequency EPR \& DFT Study, Phys. Chem. Chem. Phys. 15 (24) (2013) 9562-74. doi:10.1039/c3cp51477c. 
[9] S. Gélinas, A. Rao, A. Kumar, A. Chin, J. Clark, A. Smith, T. van der Poll, G. Bazan, R. Friend, Ultrafast long-range charge separation in organic semiconductor photovoltaic diodes, Science 343 (January) (2014) 512-517. doi:10.1126/science.1246249.

[10] A. Rao, P. C. Y. Chow, S. Gélinas, C. W. Schlenker, C.-Z. Li, H.-L. Yip, A. K.-Y. Jen, D. S. Ginger, R. H. Friend, The role of spin in the kinetic control of recombination in organic photovoltaics., Nature 500 (7463) (2013) 435-9. doi:10.1038/nature12339.

[11] A. J. Ward, A. Ruseckas, M. M. Kareem, B. Ebenhoch, L. A. Serrano, M. Al-Eid, B. Fitzpatrick, V. M. Rotello, G. Cooke, I. D. W. Samuel, The impact of driving force on electron transfer rates in photovoltaic donor-acceptor blends, Adv. Mater. 27 (15) (2015) 2496-2500. doi:10.1002/adma.201405623.

[12] J. Kudrjasova, M. Van Landeghem, T. Vangerven, J. Kesters, G. H. L. Heintges, I. Cardinaletti, R. Lenaerts, H. Penxten, P. Adriaensens, L. Lutsen, D. Vanderzande, J. Manca, E. Goovaerts, W. Maes, Designing Small Molecule Organic Solar Cells with High Open-Circuit Voltage, ChemistrySelect 2 (3) (2017) 1253-1261. doi:10.1002/slct.201601915.

[13] J. Niklas, S. Beaupré, M. Leclerc, T. Xu, L. Yu, A. Sperlich, V. Dyakonov, O. G. Poluektov, Photoinduced Dynamics of Charge Separation: From Photosynthesis to Polymer-Fullerene Bulk Heterojunctions, J. Phys. Chem. B 119 (24) (2015) 7407-7416. doi:10.1021/jp511021v.

[14] J. Behrends, A. Sperlich, A. Schnegg, T. Biskup, C. Teutloff, K. Lips, V. Dyakonov, R. Bittl, Direct detection of photoinduced charge transfer complexes in polymer fullerene blends, Phys. Rev. B 85 (12) (2012) 125206. doi:10.1103/PhysRevB.85.125206.

[15] B. Z. Tedlla, F. Zhu, M. Cox, J. Drijkoningen, J. Manca, B. Koopmans, E. Goovaerts, Understanding triplet formation pathways in bulk heterojunction polymer:fullerene photovoltaic devices, Adv. Energy Mater. 5 (2), 1401109. doi:10.1002/aenm.201401109.

[16] B. Z. Tedlla, F. Zhu, M. Cox, B. Koopmans, E. Goovaerts, Spin-dependent photophysics in polymers lightly doped with fullerene derivatives: Photoluminescence and electrically detected magnetic resonance, Phys. Rev. B 91 (8) (2015) 1-11. doi:10.1103/PhysRevB.91.085309.

[17] H. Kraus, M. C. Heiber, S. Väth, J. Kern, C. Deibel, A. Sperlich, V. Dyakonov, Analysis of Triplet Exciton Loss Pathways in PTB7:PC71BM Bulk Heterojunction Solar Cells., Sci. Rep. 6 (July) (2016) 29158. doi:10.1038/srep29158.

[18] J. Behrends, A. Schnegg, K. Lips, E. A. Thomsen, A. K. Pandey, I. D. W. Samuel, D. J. Keeble, Bipolaron formation in organic solar cells observed by pulsed electrically detected magnetic resonance, Phys. Rev. Lett. 105 (17) (2010) 12-15. doi:10.1103/PhysRevLett.105.176601.

[19] J. Gorenflot, A. Sperlich, A. Baumann, D. Rauh, A. Vasilev, C. Li, M. Baumgarten, C. Deibel, V. Dyakonov, Detailed study of N,N'-(diisopropylphenyl)-terrylene-3,4:11,12bis(dicarboximide) as electron acceptor for solar cells application, Synth. Met. 161 (23-24) (2012) 2669-2676. doi:10.1016/j.synthmet.2011.09.041.

[20] E. Thomsen, D. Keeble, B. Lochab, P. Burn, H. ElMkami, I. Samuel, Photoinduced charge separation in poly(1,4-phenylenevinylene) derivatives studied by electron paramagnetic resonance, Org. Electron. 9 (5) (2008) 809-815. doi:10.1016/j.orgel.2008.05.020.

[21] A. Konkin, C. R. Singh, S. Orlinskii, G. Mamin, A. Aganov, Light-induced $\mathrm{X}$, W-band Electron Spin Resonance study of double-crystalline donor-acceptor P3HT-b-PPerAcr block copolymers in solid thin films, Synth. Met. 215 (2016) 251-259. doi:10.1016/j.synthmet.2016.02.002.

[22] J. De Ceuster, E. Goovaerts, A. Bouwen, J. C. Hummelen, V. Dyakonov, High-frequency (95 GHz) electron paramagnetic resonance study of the photoinduced charge transfer in conjugated polymer-fullerene composites, Phys. Rev. B 64 (19) (2001) 195206. doi:10.1103/PhysRevB.64.195206.

[23] V. Krinichnyi, H.-K. Roth, A. Konkin, Multifrequency EPR study of charge transfer in poly(3alkylthiophenes), Phys. B Condens. Matter 344 (1-4) (2004) 430-435. doi:10.1016/j.physb.2003.10.024.

[24] T. Maly, T. F. Prisner, Relaxation filtered hyperfine spectroscopy (REFINE), J. Magn. Reson. 170 (1) (2004) 88-96. doi:10.1016/j.jmr.2004.06.003.

[25] T. Maly, F. MacMillan, K. Zwicker, N. KashaniPoor, U. Brandt, T. F. Prisner, Relaxation Filtered Hyperfine (REFINE) Spectroscopy: A Novel Tool for Studying Overlapping Biological Electron Paramagnetic Resonance Signals Applied to Mitochondrial Complex I, Biochemistry 43 (13) (2004) 3969-3978. doi:10.1021/bi035865e.

[26] A. Cernescu, T. Maly, T. F. Prisner, 2D-REFINE spectroscopy: Separation of overlapping hyperfine spectra, J. Magn. Reson. 192 (1) (2008) 78-84. doi:10.1016/j.jmr.2008.02.018.

[27] P. Schosseler, T. Wacker, A. Schweiger, Pulsed ELDOR detected NMR, Chem. Phys. Lett. 224 (3-4) (1994) 319-324. doi:10.1016/0009-2614(94)00548-6.

[28] D. Goldfarb, ELDOR-detected NMR,

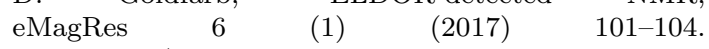
doi:10.1002/9780470034590.emrstm1516.

[29] N. Cox, A. Nalepa, W. Lubitz, A. Savitsky, ELDORdetected NMR: A general and robust method for electron-nuclear hyperfine spectroscopy?, J. Magn. Reson. 280 (2017) 63-78. doi:10.1016/j.jmr.2017.04.006.

[30] T. Hetzke, A. M. Bowen, T. F. Prisner, ELDOR-detected NMR at Q-Band, Appl. Magn. Reson.doi:10.1007/s00723-017-0927-4.

[31] C. Brabec, U. Scherf, V. Dyakonov (Eds.), Organic Photovoltaics: Materials, Device Physics, and Manufacturing Technologies, Wiley-VCH, 2014.

[32] A. Nalepa, K. Möbius, W. Lubitz, A. Savitsky, Highfield ELDOR-detected NMR study of a nitroxide radical in disordered solids: Towards characterization of heterogeneity of microenvironments in spinlabeled systems, J. Magn. Reson. 242 (2014) 203-213. doi:10.1016/j.jmr.2014.02.026.

[33] S. Stoll, A. Schweiger, EasySpin, a comprehensive software package for spectral simulation and analysis in EPR, J. Magn. Reson. 178 (1) (2006) 42-55. doi:10.1016/j.jmr.2005.08.013.

[34] F. Neese, The ORCA program system, Wiley Interdiscip. Rev. Comput. Mol. Sci. 2 (1) (2012) 73-78. doi:10.1002/wcms.81.

[35] F. Neese, Prediction of electron paramagnetic resonance g values using coupled perturbed Hartree-Fock and Kohn-Sham theory, J. Chem. Phys. 115 (24) (2001) 11080-11096. doi:10.1063/1.1419058.

[36] F. Neese, Efficient and accurate approximations to the molecular spin-orbit coupling operator and their use in molecular g-tensor calculations, J. Chem. Phys. 122 (3). doi:10.1063/1.1829047.

[37] A. D. Becke, Density-functional exchange-energy approximation with correct asymptotic behavior, Phys. Rev. A 38 (6) (1988) 3098-3100. doi:10.1103/PhysRevA.38.3098.

[38] A. Schäfer, H. Horn, R. Ahlrichs, Fully Optimized Contracted Gaussian-Basis Sets for Atoms Li to Kr, J. Chem. Phys. 97 (4) (1992) 2571-2577. 
doi:10.1063/1.463096.

[39] C. Lee, W. Yang, R. G. Parr, Development of the Colle-Salvetti correlation-energy formula into a functional of the electron density, Phys. Rev. B 37 (2) (1988) 785-789. doi:10.1103/PhysRevB.37.785.

[40] V. Barone, Structure, Magnetic Properties and Reactivities of Open-Shell Species From Density Functional and Self-Consistent Hybrid Methods, in: Recent Adv. Density Funct. Methods, Vol. 1, 1995, pp. 287-334. doi:10.1142/2914.

[41] S. Sinnecker, A. Rajendran, A. Klamt, M. Diedenhofen, F. Neese, Calculation of solvent shifts on electronic g-tensors with the conductor-like screening model (COSMO) and its self-consistent generalization to real solvents (direct COSMO-RS), J. Phys. Chem. A 110 (6) (2006) 2235-2245. doi:10.1021/jp056016z.

[42] M. Scharber, N. Sariciftci, Efficiency of bulk-heterojunction organic solar cells, Prog. Polym. Sci. 38 (12) (2013) 1929-1940. doi:10.1016/j.progpolymsci.2013.05.001.

[43] O. G. Poluektov, J. Niklas, K. L. Mardis, S. Beaupré, M. Leclerc, C. Villegas, S. Erten-Ela, J. L. Delgado, N. Martín, A. Sperlich, V. Dyakonov, Electronic Structure of Fullerene Heterodimer in Bulk-Heterojunction Blends, Adv. Energy Mater. 4 (7) (2014) 1301517. doi:10.1002/aenm.201301517.

URL http://dx.doi.org/10.1002/aenm. 201301517

[44] Y. Qin, M. A. Uddin, Y. Chen, B. Jang, K. Zhao, Z. Zheng, R. Yu, T. J. Shin, H. Y. Woo, J. Hou, Highly Efficient Fullerene-Free Polymer Solar Cells Fabricated with Polythiophene Derivative, Adv. Mater. 28 (42) (2016) 9416-9422. doi:10.1002/adma.201601803.

[45] J. S. Hyde, ENDOR of free radicals in solution, J. Chem. Phys. 43 (5) (1965) 1806-1818. doi: $10.1063 / 1.1697013$.

[46] A. Schweiger, G. Jeschke, Principles of Pulse Electron Paramagnetic Resonance, Oxford University Press, 2001.

[47] J. Kusakovskij, I. Caretti, S. Van Doorslaer, F. Callens, H. Vrielinck, Fourth stable radical species in X-irradiated solid-state sucrose, Phys. Chem. Chem. Phys. 18 (2016) 10983-10991. doi:10.1039/C6CP01118G. 